\title{
Integración de las TIC en el curso de ondas y partículas de la Universidad Autónoma de Bucaramanga
}

\author{
ICT integration in the course of waves and particles of the Universidad \\ Autónoma de Bucaramanga \\ Francy Nelly Jiménez Garcia ${ }^{1}$, Ligia Beleño Montagut ${ }^{2}$
${ }^{2}$ Departamento de matemáticas y ciencias, Universidad Autónoma de Bucaramanga, Bucaramanga, \\ Colombia \\ ${ }^{I}$ Departamento de física y matemática, Universidad Autónoma de Manizales, Departamento de Física y \\ Química, Universidad Nacional de Colombia, Manizales, Colombia \\ Correo-e: lbeleno@unab.edu.co, francy@autonoma.edu.co
}

\begin{abstract}
Resumen- El presente artículo muestra los resultados de un proyecto de investigación cuyo objetivo fue Implementar y evaluar el uso de las TIC en la enseñanza de la física en las carreras de ingeniería de la Universidad Autónoma de Bucaramanga (UNAB) usando la plataforma TEMA como metodología adicional a la tradicional. Se tomaron como muestra los resultados de los exámenes de los estudiantes de los dos semestres del 2011, y se aplicaron diversas actividades de aprendizaje con los estudiantes de los dos semestres del 2012. Para desarrollar las actividades mencionadas, la UNAB cuenta con la plataforma web MOODLE y de manera particular, en este proyecto se trata de utilizar la plataforma con nuevas actividades. El resultado obtenido de esta investigación permite trabajar de manera más cercana en las temáticas de mayor dificultad identificadas.
\end{abstract}

Palabras clave - tecnologías de información y comunicación, física, enseñanza, MOODLE.

\begin{abstract}
This article shows the results of a research project whose objective was to implement and evaluate the use of ICT in teaching physics in engineering careers at the Autonomous University of Bucaramanga (UNAB) using the TEMA platform as an additional to traditional methodology. The test scores of students in the two semesters of 2011 were sampled and various learning activities were applied with students from the two semesters of 2012. To develop these activities, the UNAB has the web platform MOODLE and particularly in this project it was use the platform with new activities. The result of this research can work more closely on the issues of greatest difficulty identified.
\end{abstract}

Key Word - Information-communication technology (ICT), physics, teaching, MOODLE.

\section{INTRODUCCIÓN}

El bajo desempeño de los estudiantes de ingeniería en asignaturas como física o matemáticas es una preocupación constante de los docentes lo cual se convierte en un problema complejo en el cual intervienen diversos factores como lo muestra el estudio realizado por Ferreyra y Gonzales, quienes plantean la necesidad de la investigación educativa en la universidad desde varias perspectivas de acuerdo a las derivaciones de su investigación [1]. Es por ello que se encuentran trabajos enfocados a mejorar dichos desempeños mediante el empleo de diversas metodologías de enseñanza que van desde el estudio de las ideas previas [2], el cambio conceptual [3], el uso de TIC [4] entre otras.

El Gobierno Nacional en su Plan Nacional de TIC 2008-2019 (PNTIC) busca que al finalizar este periodo, todos los colombianos se informen y se comuniquen haciendo uso eficiente y productivo de las TIC, [5] por lo tanto lo incluye dentro de sus ejes estratégicos de desarrollo, la Educación como uno de los ejes verticales. De esta forma, el Ministerio de Educación nacional establece los lineamientos de calidad en la educación superior, y se inicia en las universidades un proceso de reforma que contempla las horas de dedicación del estudiante, las horas de docencia directa y las horas totales por semestre, tratando de ajustarse a esos lineamientos propuestos.

Como en los demás ámbitos de actividad humana, las TIC se convierten en un instrumento cada vez más indispensable en las instituciones educativas donde pueden realizar múltiples funcionalidades [6] como son: fuente de información, canal de comunicación interpersonal y para el trabajo colaborativo y para el intercambio de información e ideas, medio de expresión y para la creación (procesadores de textos y gráficos, editores

Fecha de Recepción: 18 de febrero de 2016 Fecha de Aceptación: 15 de marzo de 2017 
de páginas web y presentaciones multimedia, cámara de vídeo); instrumento cognitivo y para procesar la información, recurso interactivo para el aprendizaje, medio lúdico y para el desarrollo psicomotor y cognitivo.

En Colombia, el Plan Nacional de TIC se estructurará alrededor una matriz de ocho grandes ejes o líneas de acción, cuatro verticales y cuatro transversales. Los ejes verticales, son aquellos que enmarcan las acciones de uso y apropiación de TIC en sectores específicos considerados críticos, uno de los cuales es la educación y se busca incorporar las TIC en el proceso educativo y de formación, para apalancar el cubrimiento y calidad. Como acciones, a desarrollar en el eje de educación, en el sector público y privado, deberán cubrir la educación básica, media y superior; la formación de alto nivel; la formación para la industria de TI; y la investigación, la vigilancia y prospectiva tecnológica y la divulgación de nuevas metodologías y técnicas para el uso de TIC en la educación. Alrededor de esta política han surgido trabajos y análisis con relación a la aplicación de las TIC en educación que contribuyen al proceso de formación [7-8]

Al respecto, desde el año 2007, la Universidad Autónoma de Bucaramanga (UNAB), de acuerdo al plan de desarrollo 20072012, incluyó formalmente dentro de los lineamientos propuestos "El uso de tecnologías de la información y las comunicaciones en pregrado y postgrado" [9], con lo cual docentes y estudiantes comienzan a involucrarse en el uso de tecnología en el aula. Posteriormente se da paso a reformas curriculares en las cuales se continúa con el énfasis de la Incorporación de TIC en el aula. El resultado presentado en este documento responde al interés de los investigadores del grupo de Investigación en Ciencias Aplicadas y docentes del departamento de Matemáticas y Ciencias Naturales por reforzar el proceso de aprendizaje para la formación del ingeniero en su ciclo básico usando otras estrategias en el proceso.

En este trabajo se hace una revisión del grado de conceptualización que adquiere el estudiante en comparación con la metodología tradicional, se identifica el nivel de satisfacción del estudiante con el uso de la plataforma TEMA, se identifican las temáticas de mayor dificultad en el curso de ondas y partículas, lo cual conlleva a plantear y usar algunas estrategias con miras a mejorar el desempeño del estudiante.

\section{METODOLOGÍA}

\section{A. Diseño de encuestas de percepción en el uso de TIC}

Se realizó una encuesta para identificar la percepción que tenían los estudiantes acerca del uso de TIC en las clases, la cual fue aplicada en el I semestre de 2012 a un total de 93 estudiantes así: 36 del curso de Ondas y Partículas, 32 del curso de Física Mecánica y 25 del curso de Electromagnetismo. Se incluyeron los estudiantes de física mecánica y de electromagnetismo ya que serían los estudiantes de los próximos cursos de ondas teniendo en cuenta que en la UNAB primero cursan física mecánica, luego electromagnetismo y seguidamente ondas y partículas.

Esta encuesta se dividió en cuatro categorías: Capacitación con 1 pregunta, Grado de satisfacción con 2 preguntas, Mejora en el desempeño con 2 preguntas y Tipo de herramientas que emplea con 8 preguntas. En esta última categoría se definieron cuatro tipos de entornos como son: portales educativos con 1 pregunta, webquest con 2 preguntas, entornos educativos con 2 preguntas y entornos colaborativos con 2 preguntas.

Se diseñó y aplicó una encuesta a docentes con el fin de conocer si creían que contaban con los materiales necesarios para hacer una clase usando TIC y si lo consideraban pertinente. Esta encuesta fue aplicada en el I semestre de 2012 a un total de 7 docentes de tiempo completo del departamento de Matemáticas y Ciencias Naturales de la UNAB.

En la encuesta para docentes se definieron cuatro categorías: Uso de Tic en el aula con 4 preguntas, habilidad con 2 preguntas, inconvenientes técnicos con 2 preguntas y creación de material con 2 preguntas.

Las encuestas se realizaron con encuestafacil.com, y el formato de los archivos es CSV el cual es utilizable en programas para el procesamiento de datos, en este trabajo se emplearon Excel y SPSS.

\section{B. Diseño de Actividades de aprendizaje con el uso de TIC para los temas de mayor dificultad en el curso de ondas y partículas}

En esta fase se identificaron los temas de mayor dificultad en el curso de ondas en los cuales se quería ofrecer como alternativa de apoyo al estudiante en su proceso de aprendizaje el uso de TIC y teniendo en cuenta que en el 2011 para estos cursos se presentó poco o ningún uso de tales tecnologías. Para tal fin, se realizó la revisión de los resultados obtenidos en las distintas evaluaciones de los cursos de ondas y partículas en los dos semestres del año 2011. Se analizaron 4 grupos en total para el 2011 así: I semestre de 2011 con dos grupos, uno del día con 23 estudiantes y uno de la noche con 25 . II de 2011 con dos grupos, uno del día con 30 estudiantes y uno de la noche con 29. Los resultados globales se muestran en la tabla 1. Aquellos temas en los cuales el porcentaje de acierto en las evaluaciones, que incluían conceptos y análisis matemático, estuvo por debajo del $70 \%$ se seleccionaron como temas de mayor dificultad.

Tabla 1. Evaluación de los temas del curso de ondas y partículas año 2011

\begin{tabular}{|l|c|c|}
\hline Tema & 2011-I & $2011-\mathrm{II}$ \\
\hline Movimiento Amortiguado & 59,5 & 50 \\
\hline Movimiento Armónico & 52 & 69,5 \\
\hline Ondas en cuerdas & 72,7 & 54,5 \\
\hline
\end{tabular}




\begin{tabular}{|l|c|c|}
\hline Intensidad sonido & 89,5 & 75,5 \\
\hline Efecto Doppler & 57,3 & 58,5 \\
\hline Snell & 75 & 74,5 \\
\hline Imágenes En lentes & 44 & 70,5 \\
\hline Efecto Compton & 67 & 72,5 \\
\hline
\end{tabular}

De acuerdo a lo anterior, los temas seleccionados para hacer incorporación de TIC fueron: movimiento amortiguado, movimiento armónico, ondas en cuerdas, efecto Doppler, y formación de imágenes. Aunque el tema de efecto Compton no logro en el I semestre de 2011 el $70 \%$ estuvo cerca y en el II semestre de 2011 si lo supero, por esta razón se dejó por fuera en cuanto a implementación de estrategias adicionales.

Paso siguiente, se diseñaron las actividades de aprendizaje para estos temas teniendo en cuenta que se contaba con el curso implementado en la plataforma MOODLE-TEMA en el cual el estudiante disponía de la información relacionada con cada temática con elementos como: el cronograma del curso, la bibliografía recomendada, presentaciones en Power Point y los laboratorios presenciales en los cuales se cuentan con una guía escrita.

A estos elementos se adicionaron talleres de estudio complementarios, chats, foros, videos demostrativos, simuladores y laboratorios virtuales usando recursos disponibles en la web, se diseñaron además guías de apoyo para laboratorios virtuales.

En cuanto a los talleres de estudio complementarios se tuvieron en cuenta las siguientes consideraciones:

- Las sesiones de clase involucraron conceptos teóricos y ejercicios, donde se hizo énfasis en la comprensión lectora. El alumno pudo consultar los documentos de TEMA durante la clase y trabajar en grupo los ejercicios bajo la dirección del profesor apoyado en el material sugerido (simulaciones, ejercicios y videos).

- Los ejercicios adicionales se consideraron de apoyo al estudiante, estos fueron desarrollados en horas fuera del horario de clase y según su disponibilidad, dando tiempo al estudiante de estudiar a su ritmo y hacerse responsable de su propio aprendizaje.

Como trabajo final se propuso a los estudiantes realizar un video sobre alguno de los laboratorios presenciales los cuales debían contener aspectos como: materiales, procedimiento, desarrollo de la práctica y cálculos más relevantes. Estos videos serían subidos a la plataforma MOODLE-TEMA para ser empleados por otros estudiantes y facilitar así el trabajo experimental. En el desarrollo de los videos los estudiantes tuvieron la oportunidad de trabajar con herramientas de edición y creación de videos e imágenes entre otras aplicaciones.

\section{Implementación de la estrategia}

Las actividades de aprendizaje diseñadas fueron implementadas en los cursos de ondas y partículas del I y II semestre del 2012 dando una continuidad a la estrategia metodológica y no solo como una implementación puntual sino como una acción continuada en el tiempo. Se tuvieron un total de estudiantes distribuidos así:

I semestre de 2012 con 2 grupos, uno del día con 34 estudiantes y uno en la noche con 13. II semestre de 2012 con 2 grupos, uno del día con 21 estudiantes y uno de la noche con 17.

Las evaluaciones de los distintos temas así como las encuestas de percepción de estudiantes y docentes fueron diseñadas por los docentes del departamento de física de la UNAB participantes en el proyecto y fueron revisadas por docentes de la Universidad Autónoma de Manizales. Se buscó realizar evaluación similares a las aplicadas en los dos semestres del 2011 para no afectar el análisis, es de anotar que las evaluaciones no eran exactamente iguales ya que se realizaron cambios en los datos o planteamientos pero siguiendo la misma intencionalidad en las preguntas.

\section{Resultados y Discusión}

A continuación se presentan los resultados de las encuestas aplicadas a docentes y a estudiantes.

\section{Encuesta aplicada a estudiantes con relación al uso de TIC}

En la figuras 1, 2 y 3 se presentan las preguntas realizadas en cada categoría y los resultados de la aplicación de las mismas al grupo de estudiantes anteriormente descrito.

\section{Categoría Capacitación:}

P1: ¿Usted ha recibido cursos o seminario sobre el uso de TIC?

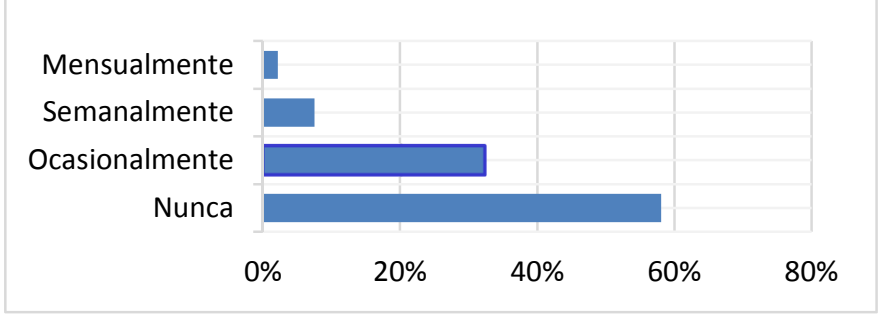

Figura 1. Resultados Categoría Capacitación

La mayoría de los estudiantes manifiestan que no han recibido capacitación en el uso de TIC o lo han recibido ocasionalmente, a pesar de ello se evidencia que logran interactuar con las TIC fácilmente gracias a que son nativos digitales; es claro que la utilización académica de estas tecnologías podría mejorar si se 
les capacitará para sacar el mejor provecho de las mismas y que no solo las emplearán como herramientas de comunicación.

\section{Categoría Grado de Satisfacción:}
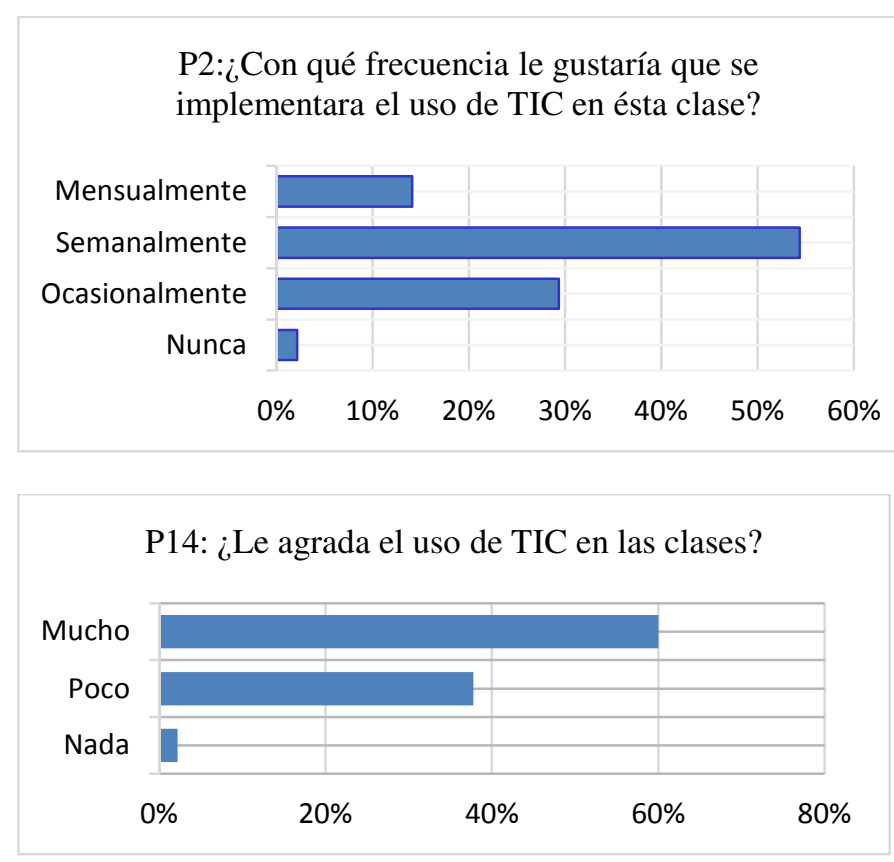

Figura 2. Resultados Categoría Grado de Satisfacción

En cuanto al grado de satisfacción con el uso de TIC en las clases se observa que más del $60 \%$ de los estudiantes encuestados les agrada que se empleen estas tecnología en las clases con frecuencias incluso semanales, frente a un $2 \%$ que manifiesta que no le agrada que se empleen estas tecnologías. Es importante realizar actividades de aprendizaje que sean del agrado del estudiantes pero en busca de acercarlos más al conocimiento científico.

\section{Categoría Mejora en el desempeño:}

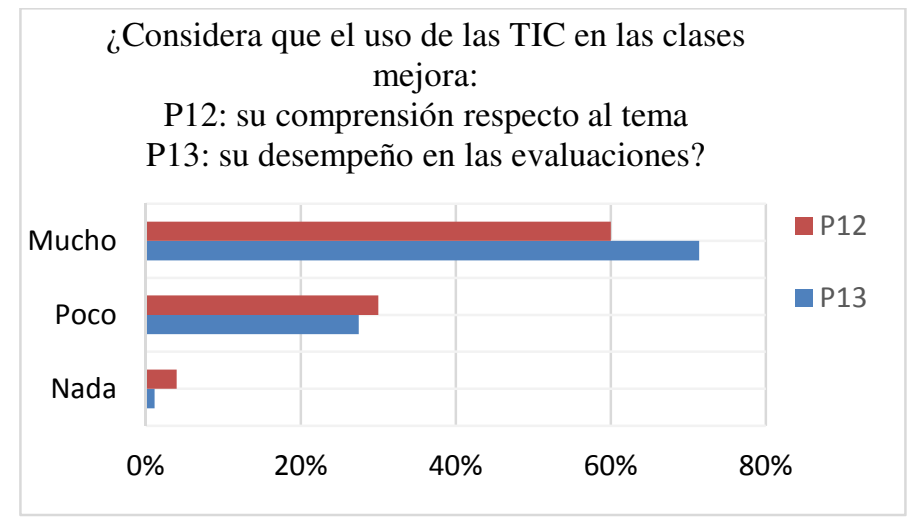

Figura 3. Resultados Categoría Mejora en el desempeño
Respecto a la mejora en su desempeño en las clases, el $60 \%$ de los estudiantes considera que mejora mucho su comprensión de los temas empleando TIC, mientras el $72 \%$ considera que mejora mucho su desempeño al prepararse con ayuda de TIC. Se presentan diferencias respecto a cada curso en particular, por ejemplo, los estudiantes de mecánica expresan que mejora su comprensión en mayor proporción (81\%) con el uso de TIC en la clase respecto a los estudiantes de electromagnetismo (52\%) y ondas y partículas $(62 \%)$.

\section{Categoría Tipo de herramientas que emplea:}

En cuanto al tipo de herramienta que emplean se encuentra que los estudiantes en general hacen uso ocasional de las mismas empleando unas más que otras, incluso, hay herramientas que nunca han utilizado y que podrían potenciar el trabajo colaborativo. En la tabla 3 se presentan los resultados respecto al uso de las distintas herramientas, las abreviaturas usadas indican: N: Nunca, O: Ocasionalmente, S: Semanalmente y M: Mensualmente.

Tabla 2. Resultados de la encuesta aplicada a estudiante sobre el uso de TIC

\begin{tabular}{|c|c|c|}
\hline Herramientas & Tipos & Resultados \\
\hline \multirow[t]{2}{*}{$\begin{array}{l}\text { Portales } \\
\text { Educativos }\end{array}$} & $\begin{array}{l}\text { P3: Páginas web: } \\
\text { Wikipedia, Icarito, } \\
\text { Wikillerato, } \\
\text { TEMA, } \\
\text { Blackboard, etc }\end{array}$ & $\begin{array}{ll}\text { N: } 0 \% & \text { O: } 24 \% \\
\text { S: } 70 \% & \text { M: } 7 \%\end{array}$ \\
\hline & P11: Blogs o wikis & $\begin{array}{ll}\mathrm{N}: 35 \% & \text { O: } 35 \% \\
\text { S: } 23 \% & \text { M: } 7 \%\end{array}$ \\
\hline \multirow[t]{3}{*}{ Web quest } & $\begin{array}{l}\text { P4:Presentaciones } \\
\text { temáticas } \\
\text { multimedia: } \\
\text { hipertexto enlaces } \\
\text { a documentos, } \\
\text { videos, podcast, } \\
\text { dibujos, imágenes, } \\
\text { simuladores, } \\
\text { Demostraciones, } \\
\text { Test, etc }\end{array}$ & $\begin{array}{ll}\mathrm{N}: 9 \% & \mathrm{O}: 42 \% \\
\mathrm{~S}: 41 \% & \mathrm{M}: 8 \%\end{array}$ \\
\hline & $\begin{array}{lr}\text { P8: } & \text { Programas } \\
\text { como } & \text { Paint, } \\
\text { Autocad, etc. }\end{array}$ & $\begin{array}{ll}\text { N: } 30 \% & \text { O: } 41 \% \\
\text { Se: } 23 \% & \text { M: } 7 \%\end{array}$ \\
\hline & $\begin{array}{l}\text { P10: Editores de } \\
\text { Video: Movie } \\
\text { Maker, Pinacle, } \\
\text { Estudio, etc }\end{array}$ & $\begin{array}{ll}\mathrm{N}: 46 \% & \mathrm{O}: 31 \% \\
\text { S: } 18 \% & \mathrm{M}: 5 \%\end{array}$ \\
\hline $\begin{array}{l}\text { Entornos } \\
\text { Educativos }\end{array}$ & $\begin{array}{l}\text { P6: Aplicaciones } \\
\text { Ofimáticas } \\
\text { Básicas: } \\
\text { procesadores de } \\
\text { Texto: Word, } \\
\text { Excel, bases de } \\
\text { datos, etc }\end{array}$ & $\begin{array}{ll}\mathrm{N}: 10 \% & \mathrm{O}: 32 \% \\
\text { S: } 52 \% & \mathrm{M}: 7 \%\end{array}$ \\
\hline
\end{tabular}




\begin{tabular}{|c|c|c|}
\hline \multirow[t]{3}{*}{$\begin{array}{l}\text { Entornos } \\
\text { colaborativos }\end{array}$} & $\begin{array}{lr}\text { P5: Usa } & \text { correo } \\
\text { electrónico } & \text { para } \\
\text { consultas } & \\
\text { específicas } & \text { del } \\
\text { curso } & \end{array}$ & $\begin{array}{ll}\mathrm{N}: 12 \% & \mathrm{O}: 44 \\
\mathrm{~S}: 32 \% & \mathrm{M}: 5 \%\end{array}$ \\
\hline & $\begin{array}{l}\text { P7: Usa chats, } \\
\text { Messenger, } \\
\text { Skype, facebook, } \\
\text { para socializar } \\
\text { contenidos del } \\
\text { curso }\end{array}$ & $\begin{array}{l}\mathrm{N}: 19 \% \\
\mathrm{~S}: 43 \% \\
\mathrm{~S}: 33 \% \\
\mathrm{M}: 5 \%\end{array}$ \\
\hline & $\begin{array}{l}\text { P9: Utiliza } \\
\text { Videocámaras y/o } \\
\text { webcams para } \\
\text { presentaciones del } \\
\text { curso }\end{array}$ & $\begin{array}{l}\mathrm{N}: 63 \% \text { O: } 23 \% \\
\text { S: } 10 \% \quad \mathrm{M}: 4 \%\end{array}$ \\
\hline
\end{tabular}

Tabla 3.Tipo de Herramienta que emplean los estudiantes

A partir del análisis global de estos resultados estadístico se considera relevante continuar con el uso de TIC con una frecuencia semanal y diversificar el tipo de actividades que puedan ser desarrolladas en forma tanto individual como grupal y dentro y fuera del aula de clase.

\section{Encuesta aplicada a docentes con relación al uso de TIC}

A continuación se presentan los resultados obtenidos en la aplicación de la encuesta a docentes desde las cuatro categorías planteadas.

\section{Categoría uso de las TIC:}

- El $86 \%$ de los docentes considera que las TIC son una herramienta de apoyo alternativa para la enseñanza de los diversos contenidos y el $14 \%$ que es un factor determinante en la enseñanza y el aprendizaje. Las opciones: es una moda de la era tecnológica en la que vivimos y es una herramienta de la cual podemos prescindir no fueron seleccionadas. Esto muestra que los docentes del Departamento de matemáticas y ciencias de la UNAB están alineados con la política de incorporación de TIC de la Universidad en la cual se concibe como una herramienta de apoyo.

- En cuanto a la frecuencia de uso que hacen en sus clases usando medios tecnológicos, el $14 \%$ opina que es alta, el $43 \%$ media, y el otro $43 \%$ baja y un $0 \%$ la opción ninguna. Esto indica que los docentes están empleando las TIC en sus clases en una proporción media baja.

- En cuanto al grado de aprovechamiento que hacen de las TIC en sus clases, el $37 \%$ consideran que este factor está por encima del $80 \%$ mientras el restante $63 \%$ consideran que tal aprovechamiento es inferior al $40 \%$.

\section{Categoría Capacitación:}

- En lo referente a la capacitación el $14 \%$ dice que su dominio de las TIC es excelente, el $72 \%$ dice que es bueno y el $14 \%$ opina que es suficiente.

- Con relación a si considera necesario tomar cursos de capacitación para el manejo de TIC para apoyar la docencia en sus asignaturas, el $57 \%$ está totalmente de acuerdo, el $29 \%$ de acuerdo, y el 14\% está en desacuerdo.

Aunque, en general, los docentes consideran tener un buen manejo de las TIC no descartan que la capacitación permanente es necesaria. Solo un $14 \%$ lo considera innecesario y coincide con aquellos docentes que dicen tener un manejo excelente de tales herramientas.

\section{Categoría aspectos técnicos:}

- El 57\% de los docentes encuestados está de acuerdo con que el aula de clase cuenta con herramientas tecnológicas para apoyo a la docencia, mientras el $43 \%$ está en desacuerdo. Lo anterior indica que aunque la UNAB ha hecho esfuerzos por dotar las aulas con herramientas tecnológicas los docentes consideran que aún falta más dotación.

- Respecto a la frecuencia con que ha presentado problemas técnicos en el momento de utilizar las TIC como apoyo a la docencia, el $14 \%$ opina que es alta, el $72 \%$ media, y el $14 \%$ baja. Muchas veces los inconvenientes técnicos desmotivan a los docentes en el uso de estas tecnologías y tales problemas presentan una ocurrencia aun considerable.

\section{Categoría creación y uso de material:}

- En cuanto a si consideran necesario que todos los temas que imparte en su asignatura requieren del apoyo de las TIC para una mejor comprensión por parte de los estudiantes, el 29\% está totalmente de acuerdo, el $43 \%$ está de acuerdo, el $14 \%$ en desacuerdo y el $14 \%$ totalmente en desacuerdo. En general los docentes encuestados consideran que la mayor parte de los temas de sus asignaturas requieren el apoyo de las TIC para lograr un mejor desempeño de sus estudiantes.

- En relación a si crean material para sus curso, el $43 \%$ de los docentes lo hacen muchas veces y el $57 \%$ pocas veces.

- El 43\% siempre usa alguna de las herramientas tecnológicas para comunicarse con sus alumnos, tales como blogs, correo electrónico, chats, foros, Plataforma TEMA, entre otras, y el $57 \%$ lo usa muchas veces.

Mientras todos los docentes expresaron emplear, con una alta frecuencia, herramientas tecnológicas de comunicación con sus estudiantes, la creación de material didáctico para sus clases es baja.

Es bueno aclarar que las TIC tienen dos partes, una de comunicación y otra de información las cuales tanto docentes y 
los estudiantes emplean con frecuencia pero es importante mencionar que la selección y diseño de actividades de aprendizaje empleando tales herramientas es otro aspecto a tener en cuenta y que requiere de más tiempo y dedicación.

\section{Implementación de la estrategia}

Los cursos del I y II semestre del año 2012 se orientaron bajo la plataforma MODLE-TEMA con las actividades de aprendizaje previamente diseñadas para cada unidad previamente seleccionada. Al finalizar cada unidad se realizaron las evaluaciones muy similares a las del año anterior, las cuales incluían uno o varios de los temas.

Tabla 3. Consolidado de los resultados de las evaluaciones para ambos años

\begin{tabular}{|c|c|c|c|c|c|c|c|c|}
\hline & $\begin{array}{c}\text { Mov. } \\
\text { Amo }\end{array}$ & $\begin{array}{c}\text { Mov. } \\
\text { Arm }\end{array}$ & $\begin{array}{c}\text { Ond } \\
\text { cuer }\end{array}$ & $\begin{array}{c}\text { Inten } \\
\text { Son }\end{array}$ & $\begin{array}{c}\text { Efe. } \\
\text { Dop }\end{array}$ & $\begin{array}{c}\text { Ley } \\
\text { Snell }\end{array}$ & $\begin{array}{c}\text { Img. } \\
\text { lentes }\end{array}$ & $\begin{array}{c}\text { Efe. } \\
\text { Com }\end{array}$ \\
\hline 2011I & 59,5 & 52 & 72,7 & 89,5 & 57,25 & 75 & 44 & 67 \\
\hline $2011 \mathrm{II}$ & 50 & 69,5 & 54,5 & 75,5 & 58,5 & 74,5 & 70,5 & 72,5 \\
\hline 2012I & 73,5 & 95,7 & 61,3 & 67,1 & 79,2 & 75 & 51,1 & 71,9 \\
\hline $2012 \mathrm{II}$ & 68,5 & 79,45 & 70,3 & 86,3 & 65,3 & 82,5 & 46,5 & 66,3 \\
\hline
\end{tabular}

Se realiza un análisis con los promedios de estos resultados para tener un consolidado entre el año sin emplear las TIC y el año empleándolas. Estos resultados se muestran en la figura 4.

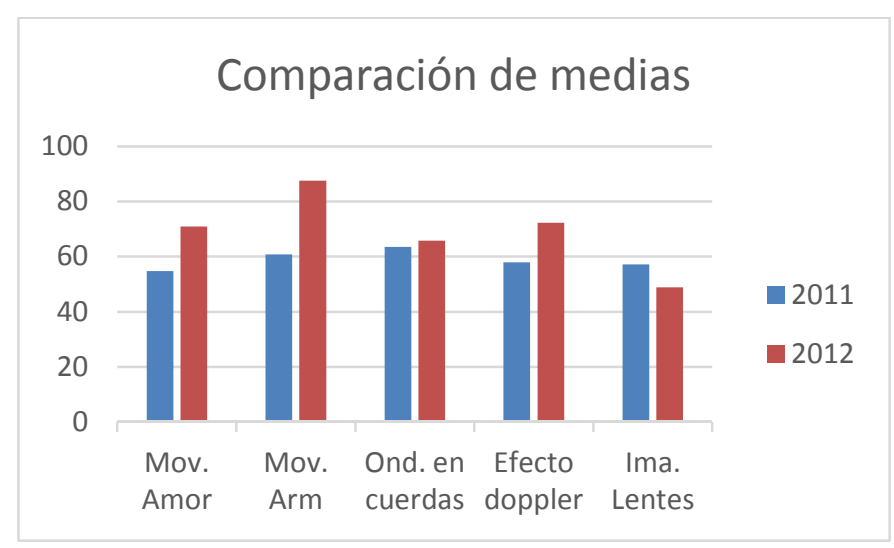

Figura 4. Comparación de los resultados obtenidos en ambos años

Se observa una evolución importante entre un año y el otro en las notas registradas para los temas movimiento amortiguado, movimiento armónico y efecto Doppler, lo cual indica que las actividades propuestas están favoreciendo el proceso de aprendizaje. Para el tema ondas en cerdas el comportamiento es similar en ambos años alcanzando apenas a superar el $60 \%$ de aprobación, lo cual indica que es necesario seguir trabajando en el diseño de actividades ya sean empleando las TIC u otra estrategia teniendo en cuenta que es un tema en el cual los estudiantes presentan dificultades para su comprensión. Para el tema imágenes en lentes hay incluso una disminución leve en el porcentaje de acierto lo cual indica que las actividades propuesta deben se rediseñadas con más detenimiento a partir de la identificación de los obstáculos de aprendizaje que presentan los estudiantes en esta temática.

Para los temas donde no se plantearon actividades diferentes a las empleadas en el 2011 el comportamiento entre un semestre y otro es muy similar, es posible que este mejore si se realizan actividades estructuras empleando las TIC.

Es importante anotar que el empleo de las TIC fue importante en este trabajo para lograr un avance en el proceso de aprendizaje pero no es la única variable a tener en cuenta, ya que entre un semestre y otro los estudiantes son diferentes y por tanto sus actitudes frente al aprendizaje también lo son. Es necesario tener en cuenta otras variables que pueden interferir en el aprendizaje en un momento dado.

\section{CONCLUSIONES}

Debido a que en la UNAB tanto docentes como estudiantes están familiarizados con la plataforma TEMA se hizo más sencillo implementar las actividades virtuales de aprendizaje diseñadas, alcanzando buenos niveles de motivación en los estudiantes frente al uso de TIC.

La implementación en el aula de test preparatorios para las evaluaciones, generó un beneficio tanto en estudiante como en el docente. En los estudiante porque pueden avanzar a su ritmo y reconocer dónde deben reforzar sus conocimientos; en el docente ya que le permite establecer los aspectos de mayor dificultad en el aprendizaje y diseñar actividades de apoyo relacionadas con dichos temas.

Fue posible emplear diferentes estrategias como: foros, chat, simuladores, páginas web existentes, entre otras, las cuales el estudiante pudo desarrollar en clase o en casa, pero contando siempre con una retroalimentadas al trabajo desarrollado.

Con relación al uso de las guías virtuales, los resultados muestran un mejor desempeño de los estudiantes cuando las actividades trabajadas usando TIC fueron desarrolladas de manera simultánea al laboratorio experimental, tal fue el caso de los temas Ley de Snell y formación de imágenes.

Los videos disponibles en la web favorecieron el aprendizaje visual y auditivo de los estudiantes puesto que lograron 
recordar fácilmente los conceptos básicos abordados en la clase. Los videos realizados por ellos contribuyeron a la práctica del trabajo en equipo y quedaron como una contribución de material para los estudiantes de próximos semestres.

El uso de foros, donde se propusieron temas de interés relacionados con tópicos especiales, fomentó en los estudiantes la importancia de estructurar una idea para presentarla a los demás, y generaron un mayor acercamiento tanto entre compañeros como con el docente.

En cuanto al rendimiento académico se logró un avance pero queda claro que el uso de las TIC no es la única variable que debe tenerse en cuenta, ya que los estudiantes entre un año y otro presentan diferentes actitudes e intereses, se deben por tanto tener en cuenta otros factores que intervienen en el aprendizaje.

\section{RECOMENDACIONES}

Se recomienda a los docentes universitarios de los cursos de física emplear las herramientas tecnológicas existentes, muchas de uso libre, y generar espacios de aprendizaje para los estudiantes diferentes al aula normal de clase.

\section{REFERENCIAS}

[1] Ferreyra, A. \& González, E. M. (2000). "Reflexiones sobre la enseñanza de la física universitaria. Enseñanza de las ciencias". Recuperado de http://www.raco.cat/index.php/ensenanza/article/viewFile $/ 21655 / 21490$

[2] Gómez, G. J. A. \& Insausti, T. M. J. (2004). Un modelo para la enseñanza de las ciencias: análisis de datos y resultados. Revista Electrónica de Enseñanza de las Ciencias, vol. 4, pp. 1-20.

[3] McDermott, L. C. (1997). Bridging the gap between teaching and learning: the role of research. AIP Conference Proceedings, vol. 399, pp. 139-165.

[4] Pontes, A. "Aplicaciones de las tecnologías de la información y de la comunicación en la educación científica". Revista Eureka sobre Enseñanza y Divulgación de las Ciencias, vol. 2, (3). Recuperado de < http://rodin.uca.es/xmlui/handle/10498/16399>

[5] Ministerio de Comunicaciones Republica de Colombia. (2008). Plan Nacional de Tecnologías de la Información y las Comunicaciones. Recuperado de $<$ http://www.eduteka.org/pdfdir/ColombiaPlanNacionalT IC.pdf $>$.

[6] Aviram, R. "Centro para el futurismo en la educación. ¿Conseguirá la educación domesticar a las TIC? Universidad Ben Gurion”. Recuperado de <http://tecnologiaedu.us.es/bibliovir/pdf/pon1.pdf>

[7] Romero, F. "Plataformas de aprendizaje sustentadas en las nuevas tecnologías de la información y la comunicación”.
Lectura y Vida: Revista Latinoamericana de Lectura, vol. 27, (4), pp. 40-47 Diciembre. 2006.

[8] Seminarios y reuniones técnicas internacionales. "Programa de Formación Avanzada a Distancia Sevilla". Tecnología de la información en el nivel de formación superior avanzada. AUIP. Salamanca. 2001

[9] Universidad Autónoma de Bucaramanga. 2007. Plan Prospectivo de Desarrollo (2007-2012). Recuperado de $<$ http://intranet.unab.edu.co/SistemaCalidad/Informacion General.jsp> 